\title{
Special issue on blockchain networks and distributed protocols
}

\author{
Maria Potop-Butucaru ${ }^{1} \cdot$ Ariel Orda $^{2} \cdot$ Maurice Herlihy $^{3} \cdot$ Stefano Secci $^{4}$ \\ Published online: 5 February 2022 \\ ( ) Institut Mines-Télécom and Springer Nature Switzerland AG 2022
}

Distributed system principles and protocols have largely influenced the way computing systems have evolved in the past 40 years. Internet applications, tactical networks, and more recently cloud computing are some examples of systems where various distributed principles can be found (e.g., mutual exclusion, leader election, consensus, synchronization, consistency, fault-tolerance).

One of the areas that has greatly benefited from the theoretical achievements in distributed computing is telecommunication systems. This happened, for instance, in the definition of routing architectures with relation to IP networks, or in the integration of distributed consensus in switching systems. On the other hand, the reliability of telecommunication networks, their ubiquitous deployment and the guarantee that a user can access the Internet anywhere and anytime, triggered the design of the Bitcoin decentralized ledger, the most prominent example of blockchain technology. As a particular form of distributed ledger technology, blockchain systems use a chain of batched on transactions (a.k.a. blocks) — as data structure-that evolves in tight coordination with the distributed validation and consensus protocols run by a very high number of dynamic nodes connected to the network.

Since the birth of Bitcoin, new cryptocurrencies are created on a daily basis, some of which still use the Bitcoin philosophy, yet many others use new data structures and original governance policies.

In this special issue, we gather seven articles spanning various aspects of blockchain technologies.

The first article, "Realizing Privacy Aspects in Blockchain Networks", authored by Oded Noam and Ori Rottenstreich, tackles the interaction between privacy and the

\footnotetext{
Maria Potop-Butucaru

maria.potop-butucaru@lip6.fr

Sorbonne University, Paris, France

Technion, Haifa, Israel

Brown University, Providence, USA

4 Cnam, Paris, France
}

public nature of the ledger information, describing challenges and novel state-of-the-art technologies to enhance privacy in blockchain networks. The conclusion of this survey is that, so far, no available technology can fully and satisfactorily meet the privacy and public ledger requirements.

Three articles then describe the application of distributed ledger technologies to telecommunication networks and the Internet of Things.

The article "Integration of Blockchain and Internet of Things: Challenges and Solutions", authored by Saima Zafar et al., studies the challenges associated to the integration of blockchain systems in IoT environments. The authors first describe the basics of blockchain systems thoroughly and then present how blockchain systems can be used to meet quality-of-service requirements of IoT devices. Future research directions in this area are described and motivated.

An application of blockchain to IoT health systems is then presented in the article "eHealthChain - A Blockchain Based Personal Health Information Management System", authored by Pravin Amrut Pawar et al. The authors propose a blockchain-based architecture for Personal Health Information Management Systems (PHIMSs), used to support operations applications working on personal health information. A four-layer architecture is proposed, where the blockchain layer is designed on top of the Hyperledger Fabric platform.

The article "BALAdIN: Truthfulness in collaborative access networks with Distributed Ledgers", authored by Vincent Messié et al., proposes a new blockchain system to support the deployment of mobile cells by local actors, such as shop tenants, in coordination with the telecommunication provider. The idea is to let the traffic conveyed by each actor be traced thanks to a blockchain-based monitoring, allowing decentralized rewarding of each actor depending on access network usage.

Two articles then present novel validation and consensus protocols for distributed ledger technologies. The article "Scalable Blockchain Execution via Parallel Block Validation", authored by Maya Leshkowitz et al., proposes a time efficient block validation approach that splits the validation 
problem into multiple disjoint tasks. The required changes to the ledge structure are presented.

The article "Voting-based probabilistic consensuses and their applications in distributed ledgers, authored " by Serguei Popov and Sebastian Mueller, describes thresholdbased voting models and discusses their possible applications for achieving consensus in cryptocurrency systems. They present the limitations in following this path as well as how these limitations can be surpassed using state-of-the-art consensus protocols.

Finally, the special issue ends with the article "Blockchain-based Whistleblowing Service to Solve the Problem of Journalistic Conflict of Interest “, authored by Antonio Emerson Barros Tomaz, José Cláudio do Nascimento and José Neuman de Souza. Whistleblowing through newspapers is common, yet it represents a particular setting where conflict of interest can have an impact on the reliability of the information and the anonymity of the sources. The authors propose a blockchain-based anonymous whistleblowing service that takes these challenges into consideration using a cryptographic scheme to ensure the whistleblower's anonymity while supporting authorship verification. Incentive compatibility is studied as well as the employment of game theory, showing how favorable conditions can be created to motivate sources to disclose a whistleblowing.

Publisher's Note Springer Nature remains neutral with regard to jurisdictional claims in published maps and institutional affiliations. 